Classification

Physics Abstracts

$33.10-33.20 \mathrm{E}$

\title{
Les constantes rotationnelles du niveau de base du méthane ${ }^{12} \mathbf{C D}_{4}$
}

\author{
M. Loete, J. C. Hilico \\ Laboratoire de Spectronomie Moléculaire (*), Université de Dijon, B.P. 138, 21004 Dijon Cedex, \\ France
}

\author{
A. Valentin, J. Chazelas et L. Henry \\ Laboratoire de Spectronomie Moléculaire (**), \\ Université Pierre-et-Marie-Curie, 75230 Paris Cedex, France
}

(Reçu le 13 novembre 1981, accepté le 15 décembre 1981)

\begin{abstract}
Résumé. - Les constantes rotationnelles $\beta_{0}, \gamma_{0}$ et $\pi_{0}$ du niveau de base de la molécule $\mathrm{CD}_{4}$ ont été déterminées à partir de l'analyse de différences de fréquences de transitions issues de niveaux de base distincts, mais atteignant le même niveau excité dans le spectre des bandes $v_{2}$ et $v_{4}$. Le spectre obtenu par transformée de Fourier a été enregistré avec une fonction d'appareil de largeur à mi-hauteur égale à $0,003 \mathrm{~cm}^{-1}$ et la précision des nombres d'ondes pour les raies simples' est égale à $\pm 0,0003 \mathrm{~cm}^{-1}$ en valeur absolue. Les constantes tensorielles ont été fixées aux valeurs déterminées par double résonance IR-RF. Les résultats obtenus sont les suivants : $\beta_{0}=B_{0}=2,632729(8) \mathrm{cm}^{-1}$;
\end{abstract}

$$
\gamma_{0}=-D_{0}=-2,7635(45) \times 10^{-5} \mathrm{~cm}^{-1} \text { et } \pi_{0}=H_{0}=0,869(90) \times 10^{-9} \mathrm{~cm}^{-1} \text {. }
$$

\begin{abstract}
The ground-state rotational constants $\beta_{0}, \gamma_{0}$ and $\pi_{0}$ have been determined for ${ }^{12} \mathrm{CD}_{4}$ from the analysis of ground-state combination-differences in the infrared spectrum of $v_{2}$ and $v_{4}$. The Fourier transform spectrum was recorded with an apparatus function of 0.003 wavenumber (FWHM) and an absolute precision of \pm 0.0003 wavenumber for unblended lines. The tensor distortion constants were held fixed at their microwave values. The results obtained are :

$$
\begin{aligned}
& \quad \beta_{0}=B_{0}=2.632729(8) \mathrm{cm}^{-1} ; \gamma_{0}=-D_{0}=-2.7635(45) \times 10^{-5} \mathrm{~cm}^{-1} \\
& \text { and } \pi_{0}=H_{0}=0.869(90) \times 10^{-9} \mathrm{~cm}^{-1} \text {. }
\end{aligned}
$$
\end{abstract}

1. Introduction. - L'analyse par extrapolations successives des spectres des différentes polyades vibrationnelles des molécules $\mathrm{XY}_{4}[1]$ suppose une détermination aussi exacte que possible des paramètres moléculaires des plus basses d'entre elles et, pour commencer, des constantes rotationnelles du niveau de base vibrationnel. En outre, une connaissance très précise (meilleure que $0,001 \mathrm{~cm}^{-1}$ ) de la structure rotationnelle de ce niveau de base est bien utile pour tester les attributions des transitions des spectres complexes des polyades supérieures. Du fait de

(*) Equipe de recherche associée au C.N.R.S., ERA 599.

(**) Equipe de recherche propre au C.N.R.S., ERA 136. 
leur symétrie, les molécules $\mathrm{XY}_{4}$ ont un moment dipolaire permanent nul ; cependant, le très faible moment dipolaire développé par distorsion centrifuge permet l'observation de transitions de rotation pure [2]. Ces transitions $Q$ obtenues par spectroscopie Stark [3] ou par double résonance IR-RF [4] permettent de déterminer les constantes tensorielles $D_{t}, H_{4 \mathrm{t}}, H_{6 \mathrm{t}} \ldots$ Il reste alors à déterminer les constantes scalaires $B_{0}, D_{0}, H_{0} \ldots$ à partir des transitions de vibration-rotation. Cette démarche, combinant les différents résultats expérimentaux, a été largement utilisée pour les molécules ${ }^{12} \mathrm{CH}_{4}$ [5], ${ }^{13} \mathrm{CH}_{4}$ [6], $\mathrm{SiH}_{4}$ [7] et $\mathrm{GeH}_{4}$ [8]. Pour la molécule $\mathrm{CD}_{4}$, les constantes scalaires $B_{0}$ et $D_{0}$ résultent de l'étude du spectre Raman de la bande $v_{3}$ [9] et, très récemment, les constantes tensorielles $D_{\mathrm{t}}, H_{4 \mathrm{t}}$ et $H_{6 \mathrm{t}}$ ont été déterminées à partir de 6 transitions radiofréquence [10]. La constante $H_{0}$ a fait l'objet d'évaluations théoriques à partir du champ de forces [11] ou de relations isotopiques [12]. Etudiant le spectre à haute résolution des bandes $v_{2}$ et $v_{4}$, que nous avons récemment enregistré, il apparaît que le modèle d'Hamiltonien d'ordre 3 utilisé nécessite une meilleure connaissance des constantes scalaires et que la qualité du spectre dont nous disposons permet une première évaluation quantitative de la constante scalaire $H_{0}$ en même temps qu'une amélioration significative de la précision de la valeur des constantes scalaires $B_{0}$ et $D_{0}$.

2. Rappels théoriques. - Au quatrième ordre d'approximation, le niveau de base d'une molécule $\mathrm{XY}_{4}$ tétraédrique s'exprime, avec les notations de Moret-Bailly [13], sous la forme :

$$
E(J, C, n)=E_{\mathrm{s}}(J)+E_{\mathrm{t}}(J, C, n) .
$$

La partie scalaire s'écrit :

$$
E_{\mathrm{s}}(J)=\beta_{0} J(J+1)+\gamma_{0} J^{2}(J+1)^{2}+\pi_{0} J^{3}(J+1)^{3},
$$

et la partie tensorielle est :

$$
\begin{aligned}
E_{\mathrm{t}}(J, C, n)=\left[\varepsilon_{0}+\rho_{0} J(J+1)\right][(2 J-3) \ldots(2 J+5)]^{1 / 2}(-1)^{J} F_{\mathrm{A}_{1} p p}^{4 J J}+ \\
+\xi_{0}[(2 J-5) \ldots(2 J+7)]^{1 / 2}(-1)^{J} F_{\mathrm{A}_{1} p p}^{6 J J}
\end{aligned}
$$

Dans la dernière formule, l'indice $p=(C, n)$ caractérise, pour $J$ donné, les différents sousniveaux rotationnels par leur symétrie $C\left(=\mathrm{A}_{1}, \mathrm{~A}_{2}, \mathrm{E}_{,} \mathrm{F}_{1}\right.$ ou $\left.\mathrm{F}_{2}\right)$ et par un numéro d'ordre $n\left(=0,1,2\right.$...). Les coefficients $F_{\mathrm{A}_{1}}^{4}$ et $F_{\mathrm{A}_{1}}^{6}$ sont des coefficients de Clebsch-Gordan du groupe des rotations adaptés à la symétrie tétraédrique [13]. Quant aux paramètres moléculaires $\beta_{0}$, $\gamma_{0} \ldots$, ils sont proportionnels aux paramètres plus « classiques » $B_{0}, D_{0} \ldots$ comme il est rappelé dans le tableau II. L'intérêt du formalisme de Moret-Bailly pour ce genre d'étude réside dans le fait que l'Hamiltonien est quasi-diagonal pour le niveau de base vibrationnel. Très précisément, au quatrième ordre, les seuls éléments non diagonaux $(e n n)$ font intervenir le seul paramètre $\xi_{0}$ et les coefficients $F_{\mathrm{A}_{1} p^{\prime}} \boldsymbol{J J}^{\prime}$, avec $n \neq n^{\prime}$; en fait leur contribution est négligeable (en regard de la précision des mesures infrarouges actuelles). Autrement dit, l'utilisation des formules (1) à (3) revient à une linéarisation du problème.

Dans le cas qui nous intéresse, les constantes tensorielles $\varepsilon_{0}, \rho_{0}$ et $\xi_{0}$ ayant été déterminées avec la précision usuelle en spectrométrie micro-onde (incertitude expérimentale de loordre de $0,03 \mathrm{MHz}$, soit $10^{-6} \mathrm{~cm}^{-1}$ ), il reste à préciser les constantes scalaires. Pratiquement, les données expérimentales sont des différences de nombres d'ondes de transitions atteignant un même niveau excité et issues de deux niveaux de base différents (transitions partenaires). Une telle différence s'écrit :

$$
v(2)-v(1)=E\left(J_{1}, C_{1}, n_{1}\right)-E\left(J_{2}, C_{2}, n_{2}\right)
$$


avec $C_{1}=C_{2}$ à cause des règles de sélection. Il est facile d'extraire la partie scalaire de ces différences :

$$
E_{\mathrm{s}}\left(J_{1}\right)-E_{\mathrm{s}}\left(J_{2}\right)=v(2)-v(1)-E_{\mathrm{t}}\left(J_{1}, C_{1}, n_{1}\right)+E_{\mathrm{t}}\left(J_{2}, C_{2}, n_{2}\right)
$$

Ces quantités sont des combinaisons linéaires des paramètres scalaires qui peuvent donc être déterminés par une méthode classique de moindres carrés.

3. Résultats. - Le spectre de $\mathrm{CD}_{4}$ a été enregistré à l'aide du nouveau spectromètre de Fourier [14] du laboratoire de Spectronomie Moléculaire de Paris dans les conditions expérimentales suivantes : température $295 \mathrm{~K}$; longueur d'absorption : $112 \mathrm{~cm}$; pression : 0,5 torr. Le domaine étudié s'étend de $930 \mathrm{~cm}^{-1}$ (région $\mathrm{P}$ de $v_{4}$ ) à $1200 \mathrm{~cm}^{-1}$ (région $\mathrm{R}$ de $v_{2}$ ). Avec l'apodisation choisie, les raies enregistrées ont une largeur à mi-hauteur comprise entre 0,005 et $0,010 \mathrm{~cm}^{-1}$ en fonction de leur intensité (rappelons que leur largeur Doppler est égale à $0,003 \mathrm{~cm}^{-1}$ ). Les deux bandes $v_{2}$ et $v_{4}$ qui apparaissent dans cette région ont été récemment étudiées par Kreiner et Robiette [15] qui ont utilisé pour le niveau de base les 3 constantes $\beta_{0}$, $\gamma_{0}$ et $\varepsilon_{0}$ alors connues. Disposant d'un spectre beaucoup mieux résolu et beaucoup plus précis, il a semblé intéressant de reprendre l'étude de ces deux bandes (qui constituent la première polyade) en utilisant la méthode décrite par Champion [16]. Cette méthode conduit à fixer les paramètres intervenant déjà dans le niveau de base, à condition bien sûr de les connaître avec une bonne précision. Par ailleurs, notre étude de $v_{2}$ et $v_{4}$ permet d'identifier avec certitude un grand nombre de transitions partenaires et d'appliquer la méthode décrite plus haut. La simplicité de cette méthode doit cependant être tempérée pour deux raisons :

a) Les transitions partenaires ont généralement des intensités très différentes (l'une étant " permise » et l'autre " interdite »). Cette observation vaut pour $v_{2}$ aussi bien que $v_{4}$ et elle limite beaucoup le choix des couples de raies.

b) Les raies des bandes $v_{2}$ et $v_{4}$ sont très souvent disposées en amas. Ceux-ci ne peuvent pas être résolus lorsque l'écart entre les raies est inférieur à leur largeur Doppler. La présence de plusieurs raies voisines limite la précision du pointé des composantes. Dans le même ordre d'idées, le spectre contient des raies dues à ${ }^{13} \mathrm{CD}_{4}$ (abondance $1 \%$ ) et des raies dues aux bandes chaudes de ${ }^{12} \mathrm{CD}_{4}\left(v_{2}+v_{4}-v_{4}\right.$ et $\left.2 v_{4}-v_{4}\right)$. Ces raies, non identifiées à ce stade de l'étude, peuvent se superposer à celles qui nous intéressent.

La détermination des partenaires nécessite donc :

- Une étude déjà bien avancée des bandes $v_{2}$ et $v_{4}$ de façon à garantir l'attribution des transitions.

- Un examen minutieux du spectre de façon à ne retenir que les raies simples et symétriques.

Pratiquement, nous avons retenu 219 paires de partenaires faisant intervenir les écarts $\Delta J=1$ ou 2, des valeurs de $J$ entre 3 et 18 et les deux bandes $v_{2}$ (96 paires) et $v_{4}$ (123 paires). La liste de ces différences figure dans le tableau I et les valeurs des paramètres obtenus dans le tableau II. L'écart-type entre les différences expérimentales figurant dans le tableau I et les différences recalculées à partir des paramètres du tableau II est de $0,00022 \mathrm{~cm}^{-1}$. Il est comparable avec la précision expérimentale du pointé des raies que l'on peut évaluer à partir du tableau I où la même différence (par exemple $(5, E, 0)-(4, E, 0))$ est obtenue à partir de plusieurs paires de transitions.

Les valeurs obtenues pour les constantes $\beta_{0}$ et $\gamma_{0}$ sont en accord avec celles publiées antérieurement [9] en ce sens que les domaines d'incertitude que nous avons déterminés sont inclus dans les précédents. Notons que les précisions données dans le tableau II résultent purement de l'analyse statistique des différences de fréquences mesurées sur un spectre unique (ce qui garantit une cohérence interne). Par contre, les précisions données par S. Brodersen et al. [9] incluent d'autres 
Tableau I. - Différences utilisées pour la détermination du niveau de base. Les différentes colonnes indiquent successivement : l'appartenance des raies à la bande $v_{2}(2)$ ou $v_{4}(4)$; l'identification de la branche $P, Q$ ou $R$ et du niveau de base par $J, C, n$ (voir texte) des deux raies $v(1)$ et $v(2)$; la différence expérimentale en $\mathrm{cm}^{-1}: \Delta_{\mathrm{obs}}=v(1)-v(2)$; l'écart en $10^{-3} \mathrm{~cm}^{-1}$ entre cette différence expérimentale et la différence recalculée $: \delta=\Delta_{\mathrm{obs}}-\Delta_{\text {calc }}$.

[Ground-state combination differences used in the determination of the ground-state constants. The entries in the different columns are : 2 or 4 when the lines belong to $v_{2}$ or $v_{4}$; assignment of each of two lines $v(1)$ and $v(2)$ as a $\mathrm{P}, \mathrm{Q}$ or $\mathrm{R}$ branch transition from the ground-state level $J, C, n$ (see text); the experimental difference $\Delta_{\text {obs }}=v(1)-v(2)$ in $\mathrm{cm}^{-1}$; the residual $\delta=\Delta_{\text {obs }}-\Delta_{\text {calc }}$ in $10^{-3} \mathrm{~cm}^{-1}$.]

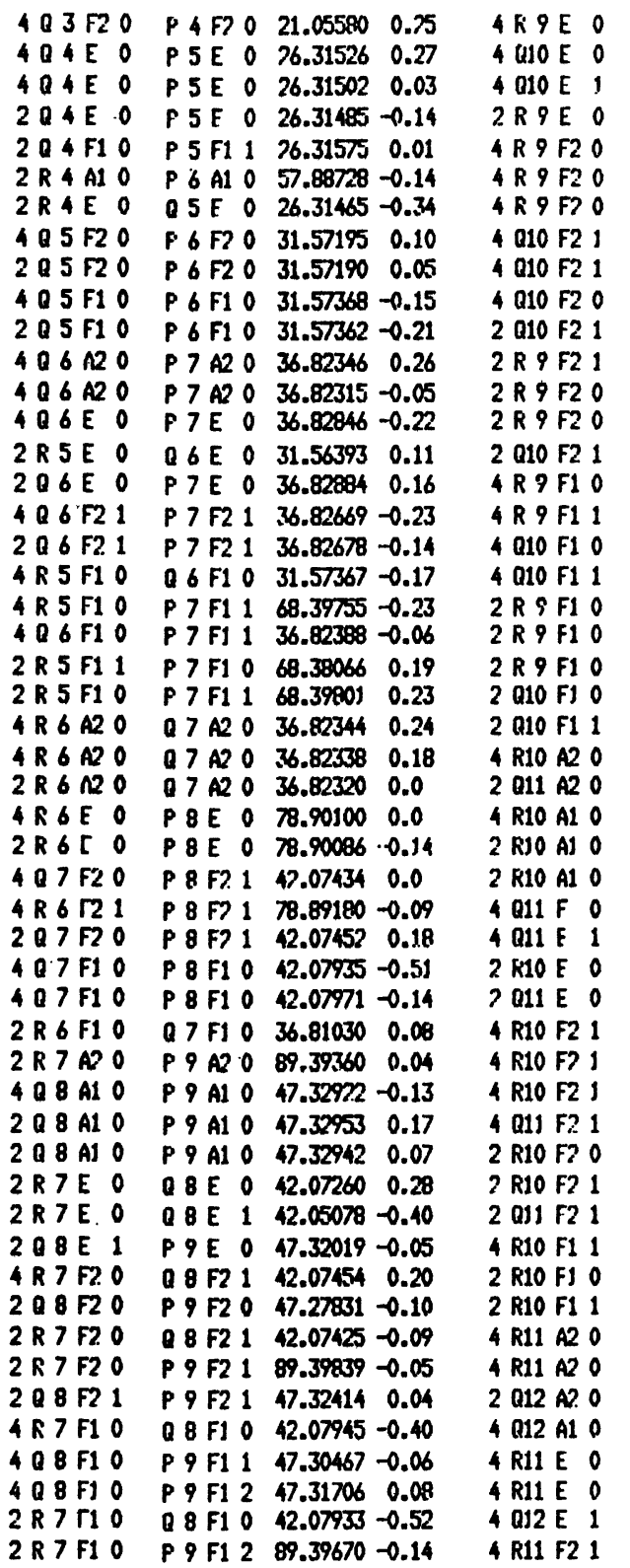

P1) E $1110.35330-0.15$ P1I E $1 \quad 57.78600 \cdots 0.12$ P11 E $0 \quad 57.80310 \quad 0.36$ $\begin{array}{llll}\text { P11 F } 1 & 110.35365 & 0.20\end{array}$ 010 F2 I $52.56160-0.10$ Q10 F2 I $52.56148-0.22$ P11 F2 2 110.367.5 0.13 $\begin{array}{lllllll}\text { P11 F2 } 2 & 57.80608 & 0.35\end{array}$ P11 F7 ? $57.80560-0.12$ P1) F2 I $57.74219-0.34$ P11 F2 $1 \quad 57.7710 .3 \quad 0.04$ $\begin{array}{lllll}010 & F 2 & 1 & 52.52597 & 0.03\end{array}$ Q10 F7. $1 \quad 52.56165-0.06$ P11 F2 2 110.36723 -0.19 P11 r2 $2 \quad 57.80557-0.15$ P11 FI 1 110.35324 -0.12 . P11 F1 $2110.35445 \quad 0.15$ P11 F1 $1 \quad 57.76898 \quad 0.16$ $\begin{array}{llllllll}\text { F11 F1 } & 57.78737 & 0.03\end{array}$ $\begin{array}{llll}\text { 0JO F1 } 0 & 52.58495 & 0.30\end{array}$

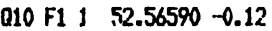
$\begin{array}{llllllllll}\text { P11 F1 } 1 & 110.35341 & 0.05\end{array}$ P11 F1 $1 \quad 57.76845 \quad-0.27$ $\begin{array}{lllllll}\text { P11 F1 } & 57.78 / 51 & 0.17\end{array}$ $\begin{array}{llllllllll}02 & 0 & 57.79607 & 0.26\end{array}$ P12. A2 $0 \quad 63.04503-0.04$ $\begin{array}{llllllll}P 12 & \mathrm{Al} & 0 & 120.81303 & 0.01\end{array}$ F12. A1 $1120.68993 \quad 0.33$ PJ? A1 $0 \quad 320.813190 .16$ P12 I. $0 \quad 63.01591 \quad 0.17$ $\begin{array}{llll}P 121 & 0 & 62.97794 & 0.13\end{array}$ Q11 E $0 \quad 57.74773-0.44$ P12 E. $0 \quad 63.015980 .14$ P12 $220120.81894-0.29$ $\begin{array}{llll}\text { Q11 } & \text { I? } 1 & 57.77109 & 0.10\end{array}$ P12 F2 I $120.79247-0.24$ P12 Г?: $163.02138-0.34$ $\begin{array}{llllll}\text { Q11 } & \text { 2 } & 57.74265 & 0.12\end{array}$ $\begin{array}{lllll}\text { P12 F2 } 0 & 120.81949 & 0.26\end{array}$ P1) F7 $1 \quad 63.02193 \quad 0.21$ P12 F1 $0120.81926-0.35$ R11 F1, $57.76866 \cdot 0.06$ P12 F1 0 120.81941 -0.20 012 A2 P $43.04503-0.04$ P13 A2 $0131.253 \% 8$ 0.04 P13 A2 O $68.20798-0.19$ P13 A1 $0 \quad 68.13680-0.02$ Q12 E $0 \quad 63.01556-0.18$ P13 E $1131.27652-0.23$ P13 E $0 \quad 68.25325-0.40$ 012 F2 $\quad 63.02226 \quad 0.55$
4 RI, AI 0

4 RI3 [ $O$

4 PIS E 1

4 OIS F ?

2 OS4 E. 1

$4 \mathrm{R} 13 \mathrm{FI}$ ?

4014 FIO

$2014 F 11$

4015 F 0

4 QJ5 F2 O

4 R14 F2?

4 RI4FI 1

4 R14 F1 2

4 R15 A2 0

$4016 \mathrm{E}$ ?

4 R15 F2 ?

4 RIS F2 1

$4016 \mathrm{F1} ?$

4 R15 F1 1

4 R16 E 1

4 R16 F2 3

4 R16 FI 2

$4 \mathrm{R} 17 \mathrm{FI}$ ?

$2 R 4$ Al $O$

$206 E 0$

$4 Q>E 0$

$2 Q 8 F>0$

AR\&F?

2 I: 9 A2 0

$4 R 9 F 21$

$4 k 9+2.1$

4010 F? 0

2 K1O A2 0

2 R1O A? $O$

4 RJOE 1

4 RIO E 1

4 RIO E I

2 RiO F. 1

2 R1O F 1

4 K1O F2?

4 RIO F?

4 R1O FI

4 K1O F1O

4 R1O FI 1

4011 F1 2

2. R11 A2 O

2 RJ1 A2 0

2. $\mathrm{R} 12 \mathrm{~A} 2 \mathrm{O}$

2 R11 $\mathrm{F} 2 \mathrm{O}$

2 R11 F2 O

2 R11 F2 1
(1)4 Al $0 \quad 73.44737 \quad 0.17$ $\begin{array}{llllll} & 114 \mathrm{~F} & 1 & 73.46947 & 0.20\end{array}$ H'15 F, $70.64399-0.21$ P15 E 0 78.68652 -0.03 FIE E $178.64375-0.35$ 014 F1 0 73.48445 0.06 $\begin{array}{lllll}\text { P.15 } \Gamma 12 & 79.59271 & 0.63\end{array}$ $\begin{array}{lllll}P 15 & F 1 & 2 & 78.15727 & 0.14\end{array}$ P16. E $1823.86546 \quad 0.39$ P16 F2 $383.88739-0.17$ 015 F2 $278.67915-0.03$ $\begin{array}{llllllllll}015 & F 1 & 2 & 78.65741 & 0.28\end{array}$ $\begin{array}{llllll}015 & F 1 & 1 & 78.66245 & -0.03\end{array}$

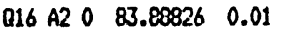
$\begin{array}{lllll}P 17 & 0 & 89.08336 & -0.24\end{array}$ Q16 F2 $183.84357-0.16$ 016 F2 $283.87129-0.10$ $\begin{array}{llll}\text { P17 FI } 1 & 89.08467 & 0.13\end{array}$ 016 F1 $383.87054-0.24$ $\begin{array}{llll}017 & 1 & 89.06735 & -0.33\end{array}$ 017 F2 389.071430 .06 017 F1 $2 \quad 89.05974-0.02$ $\begin{array}{lllll}018 & F 1 & 1 & 94.25388 & 0.01\end{array}$ P 6 A1 $0 \quad 57.83728-0.14$ $\begin{array}{lllll}P \text { ᄀ E } & 0 & 36.82919 & 0.51\end{array}$ $P 8 E \quad 1$ 22.05164 0.46 P Q F2. $O \quad 47.27831-0.10$ ['10 F? O $99.87899 \quad 0.40$ H11 A2 0 110.29141 -0.01 R1O F2 0 52.55431 -0.10 P11 F\%, I 110.29649 - 0.44 P11 F2 $15.74336 \cdot 0.17$ Q11 A2 O $5 \% .79542-0.18$ F'17 A2 O 120.840k5 - 0.72 R11 F $0 \quad 5 \% .602890 .15$ Q11 $\Gamma$ I 157.840890 .20 F1? E 0 120.8188\% 0.33 (11) $F \quad 0 \quad 57.80273 \quad 0.0$

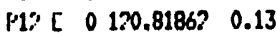
Q11 12 157.800570 .46 P17 F2 I $120.82195 \quad 0.12$ Q11 FI ? $57.79152 \quad 0.02$ P12 F1 0 120.80075 -0.24 $\begin{array}{llllll}\text { R11 FI } 2 & 57.81017 & 0.0\end{array}$ P12 F1 $0 \quad 63.00923-0.26$ $\begin{array}{llllll}012 & \text { A2 } & 6 & 63.04510 & 0.03\end{array}$ F13 A2 $0 \quad 131.25308-0.16$ P13 A2 $0 \quad 68.20799-0.19$ Q17 F2 $263.02856-0.03$ P13 F) \& $131.27951-0.11$ $\begin{array}{llllll}\text { F13 F2 } 2131.27463 & 0.0\end{array}$ 
Tableau I (suite).

\begin{tabular}{|c|c|c|c|}
\hline 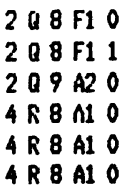 & $\begin{array}{llll}\text { P } & 9 & \text { F1 } & 2 \\
\text { P } 9 & \text { F1 } & 1 \\
\text { P10 } & \text { A2 } & 0 \\
\text { Q } 9 & \text { A1 } & 0 \\
\text { Q } 9 & \text { A1 } & 0 \\
\text { P1O A1 } & 0\end{array}$ & $\begin{array}{l}47.31737 \\
47.32224 \\
52.49585 \\
47.32959 \\
47.37930 \\
99.87690\end{array}$ & $\begin{array}{r}0.38 \\
-0.03 \\
0.23 \\
0.23 \\
-0.06 \\
-0.03\end{array}$ \\
\hline & PJO A1 0 & 52.54750 & 0.03 \\
\hline $409 E 0$ & $\begin{array}{l}\text { P10 A1 } 0 \\
\text { P10 E } 0\end{array}$ & $\begin{array}{l}52.54768 \\
52.56734\end{array}$ & $\begin{array}{r}0.21 \\
-0.01\end{array}$ \\
\hline & $\begin{array}{ll}\text { Q } 9 \text { E } & 0 \\
\text { P10 F2 } & 1\end{array}$ & $\begin{array}{l}47.29910 \\
52.56203\end{array}$ & $\begin{array}{l}0.0 \\
0.33\end{array}$ \\
\hline F2O & P1O F2 1 & 52.56168 & -0.03 \\
\hline & & 99.87871 & $\begin{array}{r}0.20 \\
-0.34\end{array}$ \\
\hline & $\begin{array}{l}\text { P10 F1 } 1 \\
\text { P1O F1 } 1\end{array}$ & $\begin{array}{l}52.56596 \\
52.54396\end{array}$ & $\begin{array}{l}-0.06 \\
-0.21\end{array}$ \\
\hline & P10 F1 1 & 52.53198 & 0.06 \\
\hline & Q $9 F 11$ & 47.30475 & 0.02 \\
\hline & P10 F1 1 & 52.56624 & 0.21 \\
\hline $\begin{array}{l}2010 \mathrm{~A} 2 \\
2 \mathrm{R} 9 \mathrm{A1}\end{array}$ & $\begin{array}{l}\text { P11 A2 } 0 \\
\text { Q10 A1 } 0\end{array}$ & $\begin{array}{l}57.79576 \\
52.54755\end{array}$ & $\begin{array}{r}-0.04 \\
0.07\end{array}$ \\
\hline $4 \times 9 F 0$ & Q10 E. 0 & 52.56731 & -0.04 \\
\hline
\end{tabular}

4 RI1 F? 1

4 RII F2 1

4 Q12 F2 1

2 Q12 F2?

4 R11 F1 1

4012 F1 2

2 RII F1 2

2012 F1 1

4 R12 A2 O

4 RJ2 A1 1

2 RI3 AI 0

4 R12 F2 I

4 R12 F? ?

4 K12 F1 1

$4 \mathrm{K12} F 1$

4 R12 F1 1

4 k12. FJ 1

4013 F1?

4013 F1 1

2013 F1 2

4014 A2 0

4 R13 A1 O $\begin{array}{llll}012 \text { F2 } 1 \quad 63.02176 & 0.04\end{array}$ P13 F2 2 131.27466 0.03 P13 F2 $168.215 P 8-0.01$ P13 F2 $168.25095 \cdot 0.09$ P13 F1 $3131.26378 \quad 0.25$ P13 F1, $\$ 8.255660 .07$ $\begin{array}{llllll}\text { Q12 F1 } 1 \quad 62.94877 & 0.43\end{array}$ P13 F1 ? $68.7 .4994 \quad 0.15$ Q13 A2 $0 \quad 68.20815-0.03$ 013 A) $0 \quad 68.25 .984-0.36$ P14 A1 0 73.44746 0.26 P14 F2 $0141.70146-0.46$ P14 F2, $141.69079-0.18$ 013 F1 ? $18.238553-0.26$ Q17. F1 $2 \quad k 8.27848-0.31$ $\begin{array}{llll}\text { llis } & \text { If } 2 & 68.23898 & 0.19\end{array}$ $\begin{array}{lllllll}P 14 & 11 & 0 & 141.72337 & 0.20\end{array}$

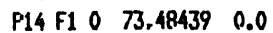
$P 1411173.46109 \cdot 0.21$ $\begin{array}{lllll}P 14 & F 1 & 0 & 73.48441 & 0.02\end{array}$

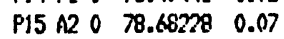
O14 A1 $073.44736 \quad 0.15$
2 RJ1 F2 2

A RII F2 1

2. kill $F 10$

2 Ri1 F) 0

2 R11 11

2 R12 A2 0

2 K12 AI 0

2 R12 A1 1

$2 \mathrm{RJ2}$ AJ 1 .

? RI2 F2 0

7 Fi12 F2 2

$2 \mathrm{RJ} 2 \mathrm{~F} 1 \mathrm{I}$

2 R12 F1

( R12 |1 I

2 RI3 A1 O

4 R13 FI ?

2. 015 A1 0

2.R14 F 0

2 R14 F2 0

4 R14 F2 2

4 K14 F10

4 RIS F2 2
012 F2 $1 \quad 62.98705 \quad 0.06$ Q12 F7 $0 \quad 63.04803-0.20$ Q12. F1 $1 \quad 63.03345 \quad 0.14$ P'13 F1 2 131.27258 0.29 P13 F1 $3131.26338-0.14$ 013 A2 $0 \quad 68.20807-0.10$ Q13 AJ $0 \quad 88.13678-0.03$ 013 AJ $0 \quad 18.75990-0.34$ P14 As $0141.70738-0.07$ 013 521 K $1 . .18922-0.15$ P14 F2 $1141.69046-0.01$ 013 FI $768.23053-0.26$ P14 F1 O 161.7229? -0.26 013 F1 $1 \quad 68.19658$ - 0.24 (114 A1 $0 \quad 73.44759 \quad 0.38$ R14 FI $173.41937 \quad 0.04$ P16 AJ 0 83.78407 0.13 U15 E , 78.57985, 0.59 Q15 F2 ? $78.54248 \quad 0.20$ Q15 F? $2 \quad 78.67917-0.0$ ? $\begin{array}{lllll}015 & F 1 & 2 & 78.59259 & 0.49\end{array}$ Q16́ F2 $183.84 .352-0.16$

Tableau II. - Les constantes du niveau de base de ${ }^{12} \mathrm{CD}_{4}$. Les valeurs sont exprimées en $\mathrm{cm}^{-1}$. Les incertitudes sont notées entre parenthèses dans l'unité du dernier chiffre qui précède. Pour la première colonne l'incertitude vaut 2,5 écart-type (intervalle de confiance à $99 \%$. Pour la seconde colonne, les valeurs et les incertitudes sont tirées des références [9] pour $\beta_{0}$ et $\gamma_{0},[11,12]$ pour $\pi_{0}$ et $[10]$ pour $\varepsilon_{0}, \rho_{0}, \xi_{0}$.

[The ground-state constants of ${ }^{12} \mathrm{CD}_{4}$. The values are in $\mathrm{cm}^{-1}$. The uncertainties are in units of the last quoted decimal place. In the first column, errors are 2.5 standard deviations $(99 \%$ confidence intervals). In the second column, values and errors are taken from references [9] in the case of $\beta_{0}$ and $\gamma_{0}$, from $[11,12]$ in the case of $\pi_{0}$ and from [10] in the case of $\varepsilon_{0}, \rho_{0}, \xi_{0}$.]

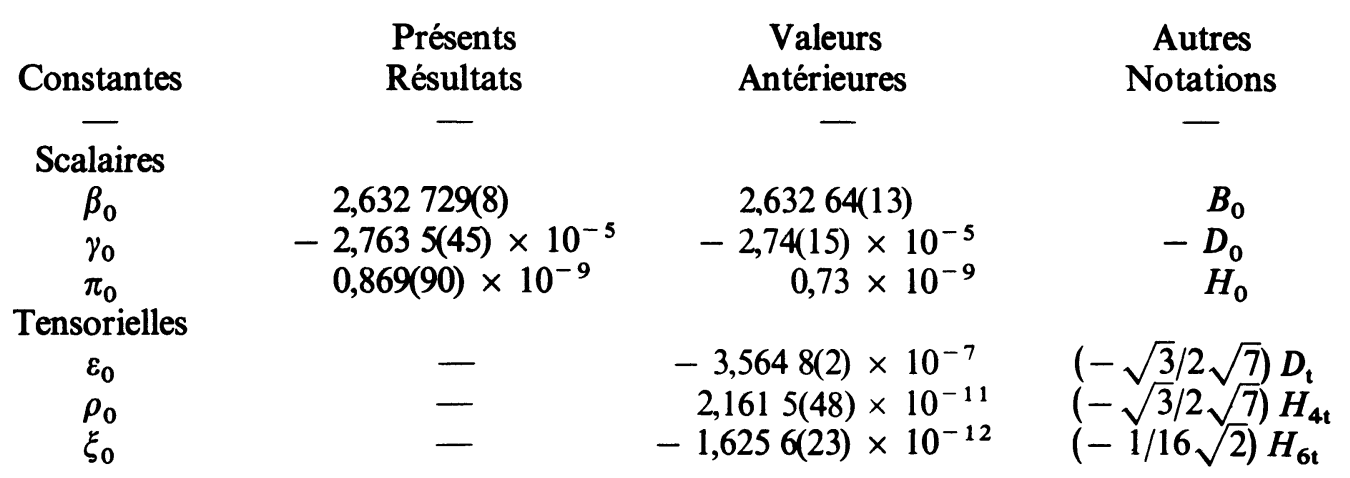

sources possibles d'erreurs liées à la méthode utilisée par ces auteurs : leur détermination de $B_{0}$ et $D_{0}$ résulte indirectement d'une analyse simultanée des spectres Raman et infrarouge de la bande $v_{3}$; elle implique, par nature, des corrélations importantes avec les constantes du niveau excité.

4. Conclusion. - Ce travail conduit à une amélioration significative de la connaissance des constantes scalaires du niveau de base de $\mathrm{CD}_{4}$ et, en particulier, à une première détermination 
expérimentale de la constante $\pi_{0}=H_{0}$. Avec les constantes tensorielles déjà connues, il permet de calculer le niveau de base avec une précision de $0,0002 \mathrm{~cm}^{-1}$ c'est-à-dire du même ordre de grandeur que celle des nombres d'ondes sur les spectres actuellement disponibles. Ces valeurs permettent un calcul précis de différences entre niveaux de base, en vue de l'étude des spectres infrarouges. Elles permettent aussi d'utiliser avec une plus grande efficacité, une méthode d'extrapolation, pour l'étude des polyades supérieures et, pour commencer, la diade $v_{2}$ et $v_{4}$ que nous avons obtenue.

\section{Bibliographie}

[1] Champion, J. P. et Pierre, G., J. Mol. Spectrosc. 79 (1980) 255-280.

[2] Watson, J. K. G., J. Mol. Spectrosc. 40 (1971) 536-544.

[3] OzIer, I., Phys. Rev. Lett. 27 (1971) 1329-1332.

[4] Curl Jr., R. F. and OKa, T., J. Chem. Phys. 58 (1973) 4908-4911.

[5] Tarrago, G., Dang-Nhu, M., Poussigue, G., Guelachvili, G. and Amiot, C., J. Mol. Spectrosc. 57 (1975) 246-263.

[6] Pinkley, L. W., Narahari Rao, N., Dang-Nhu, M., Tarrago, G. and Poussigue, G., J. Mol. Spectrosc. 63 (1976) 402-444.

[7] Ozier, I., Lees, R. M. and Gerry, M. C. L., Can. J. Phys. 54 (1976) 1094-1105.

[8] Kreiner, W. A., Opferkuch, R., Robiette, A. G. and Turner, P. H., J. Mol. Spectrosc. 85 (1981) 442-448.

[9] Brodersen, S., Gray, D. L. and Robiette, A. G., Mol. Phys. 34 (1977) 617-628.

[10] Kreiner, W. A. and Robiette, A. G., J. Chem. Phys. 74 (1981) 3713-3717.

[11] Gray, D. L. and Robiette, A. G., Mol. Phys. 37 (1979) 1901-1920.

[12] Watson, J. K. G., J. Mol. Spectrosc. 74 (1979) 483-485.

[13] Moret-Bailly, J., J. Mol. Spectrosc. 15 (1965) 355-377.

[14] Valentin, A., Connes, P. et Henry, L., Appl. Opt., à paraître.

[15] Kreiner, W. A. and Robiette, A. G., Can. J. Phys. 57 (1979) 1969-1981.

[16] Champion, J. P., Can. J. Phys. 55 (1977) 1802-1828.

Commission paritaire $N^{\circ} 59.024$

(C) Editions de Physique 1982

Directrice de la Publication : Jeanne BERGER

Imprimé en France. - Imprimerie JOUVE, 18, rue Saint-Denis, 75001 PARIS

Dépôt légal : Février 1982 